\title{
Euthanasia is not an option: 10 years' care of a chimpanzee with acute tetraparesis
}

\author{
Tetsuro Matsuzawa ${ }^{1,2,3}$
}

Published online: 11 June 2016

(C) Japan Monkey Centre and Springer Japan 2016

We have been taking care of a male chimpanzee at the Primate Research Institute, Kyoto University, since he became completely paralyzed from the neck down after the onset of acute tetraparesis. Almost 10 years have passed since we began to look after this disabled chimpanzee. Three additional papers report on this case (Miyabe et al. 2010; Hayashi et al. 2013; Sakuraba et al. 2016). The most recent of these articles (Sakuraba et al. 2016) has just been published in Primates. For the first 14 months, the chimpanzee was completely immobile, lying on his back. He then gradually recovered enough function to sit up; using suspended ropes he was able to pull himself upright by his arms. Following physical therapy and the use of a novel computer-assisted rehabilitation program, he can now walk unaided. This specially designed innovative system to facilitate walking involves a touch-screen cognitive task. I want to take this opportunity to give a brief account of this rehabilitation.

On the morning of 16 September 2006, a 24-year-old male chimpanzee named Reo was found lying immobile on the ground. Please take a look at his personal record (GAIN registration number 0439) in the GAIN database (Matsuzawa 2016): see http://shigen.nig.ac.jp/gain/. Reo is a member of the community of 13 chimpanzees at the Primate Research Institute, living in a semi-natural outdoor compound attached to an indoor facility (Matsuzawa et al. 2006). Following examination using a variety of different

Tetsuro Matsuzawa

matsuzawa.tetsuro.8w@kyoto-u.ac.jp

Institute of Advanced Study, Kyoto University, Kyoto, Japan

2 Primate Research Institute, Kyoto University, Inuyama, Japan

3 Japan Monkey Centre, Inuyama, Japan techniques, including functional magnetic resonance imaging, we found that the chimpanzee's condition most closely resembled that of acute transverse myelitis in humans. A portion of the spinal cord in the neck had become narrowed. The chimpanzee's face and head were fully functional, but below the neck the body was completely paralyzed.

The young researchers, care staff, and veterinarians of the institute spontaneously organized themselves into a bold and enterprising team. Between them, they created a comprehensive rota, to take care of the chimpanzee $24 \mathrm{~h} /$ day. Thus there was always someone at hand to help Reo. Veterinarians adopted various methods to suppress Reo's inflammation, including steroid therapy and intravenous drip infusion. The 24 -h care system continued for about 2 months, until 30 November 2006.

Lying on a bed for a prolonged period causes pressure ulcers. Volunteer staff devised a solution for these sores: a soft pliable bed and a hanging apparatus, allowing carers to raise the chimpanzee's limbs, which reduced pressure on his back. Although the condition of the skin on his back improved, pressure ulcers then developed on Reo's elbows and knees. The skin broke and bled. Reo's body weight dropped to a low of $35.0 \mathrm{~kg}$ from an initial weight of $57.4 \mathrm{~kg}$. By 18 December 2006, forty percent of Reo's original body weight had been lost. Reo's physical appearance had also deteriorated (see Fig. 1). He had become very thin, and parts of his skin bled and suppurated due to the pressure ulcers. In short, Reo looked very miserable (Miyabe et al. 2010).

Given a similar situation in Europe and North America, euthanasia would most likely have been considered the correct course of action, due to the belief that it is not good for animals to experience pain and suffering. Other problems include the cost in terms of time of labor- 


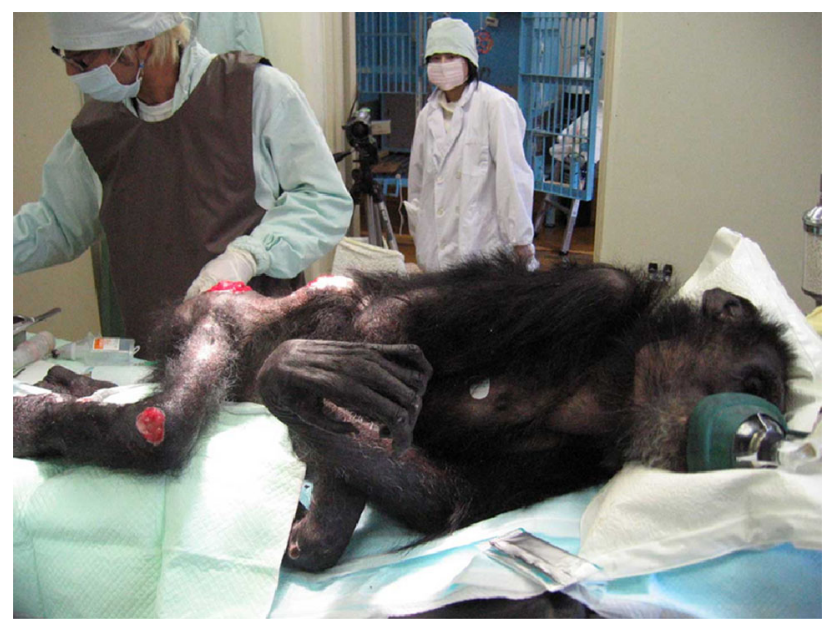

Fig. 1 Deteriorated physical appearance of male chimpanzee, Reo, following the onset of acute tetraparesis

intensive care, along with chronic fatigue experienced by care staff, and the high financial cost of medication (no health insurance being provided for the care of chimpanzees). In truth, I heard hushed voices asking me, as the director of the institute at that time, "How long will you continue to take care of this chimpanzee?" However, not one of the volunteer carers even considered euthanasia as an option. This may, in part, be due to Japanese cultural and religious beliefs, rooted in Buddhism: we should kill no living creatures. However, the most important contributing factor was Reo's demeanor. Even during the most difficult period, he never seemed to show signs of depression.

At peak health and completely mobile, Reo seemed to derive pleasure from frequently teasing caretakers and students. Young male chimpanzees often display dominance towards humans through mock aggression. Reo had been known to be a very playful young chimpanzee. However, even under almost total paralysis, he still managed to express this side of his personality. Lying immobile on his back, function remained intact above the neck, so that he was able to drink liquids through a straw from containers. He would pretend to drink water, but hold it in his mouth. When a young person approached him to carry out care tasks, he would suddenly spray them with water. The carers were caught completely by surprise, and Reo smiled. In this sense, nothing had changed between before and after the onset of his illness. Despite the restriction in movement, he was fully "alive" and still "himself." He appeared to be reasonably satisfied with the situation. Supposing that I had been in Reo's situation, I might well have been anxious about my future and become depressed. However, Reo did not show any signs of depression. Chimpanzees seem to live in the world of here and now.
Thankfully, by the fourteenth month after the onset of paralysis, Reo had begun to sit up (Hayashi et al. 2013). He then progressively regained the ability to move his neck, shoulders and finally his upper limbs. He became able to grab hold of suspended rubber ropes and pull his body upright into a sitting position (see Fig. 2). So we moved Reo from a cage to a large room where he could move by brachiation, using his upper limbs. Next, we introduced a program of physical therapy. Two young members of staff, a veterinarian and a caretaker, became Reo's closest human contacts through a period of intensive daily care. They entered the cage with Reo and acted in a playful manner towards him: tickling, scratching, pushing, holding etc. Reo particularly loved to be groomed, especially at the back of his ears. One person would play with and tickle Reo while the other tried to extend his contracted legs. It appeared to be painful. Reo seemed to dislike seeing his flexed legs extended. However, because of the play context he began to accept this physical therapy. He gradually got much better. His behavioral recovery over a 41-month period is described in the second paper (Hayashi et al. 2013). Eventually, he was able to climb and brachiate using his arms. The remaining challenge was for him to walk across the floor by himself.

Prior to the paralysis, Reo had experience of doing cognitive tasks on a touch screen. To facilitate spontaneous walking, we introduced a modified computer-based cognitive task. The set up comprised a computer-controlled monitor attached to one wall. Reo was accustomed to receiving a reward of a small piece of food each time he

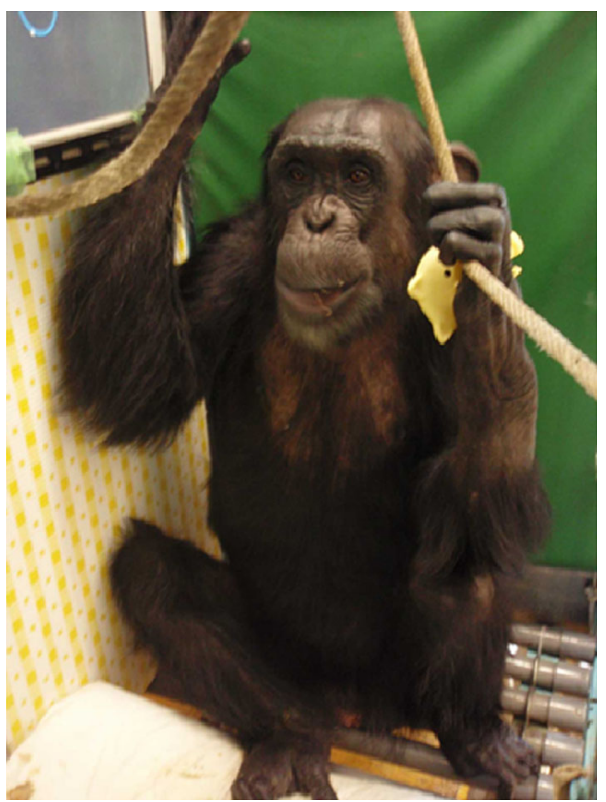

Fig. 2 Reo sitting up by grasping ropes after lying on his back for 14 months following the onset of acute tetraparesis 
succeeded in carrying out the presented task. However, now the food reward was delivered via a food tray attached to the wall opposite the touch screen. This required Reo to walk a minimum of $2 \mathrm{~m}$ to reach the reward. In order to return to the monitor, to start the next task, he then had to make the 2-m return journey. Thus, between each successive food reward, he had to walk a distance of $4 \mathrm{~m}$. Participation was completely voluntary. Furthermore, the total amount of food per day was strictly controlled, so that, regardless of Reo's performance of the tasks, he received the same total amount of calories overall. He did not have to wait until a specific time, breakfast, lunch or dinner, to eat. Food was freely available. Given the option, Reo preferred to work for food, by solving the problem and walking. Each session comprised 100 tasks, and he received two sessions per day. On average, he walked $800 \mathrm{~m}$ in a single day. Thus, $8 \mathrm{~km}$ over 10 days and $80 \mathrm{~km}$ over 100 days.

At first, Reo used a rope, attached to the wall, to move. He held onto the rope to pull himself forwards, his immobile legs sliding across the floor. He then developed a "crutch walk," placing his fists on the ground to move himself forward. Finally, without the aid of his hands, Reo started moving in an upright seated position, in a side-to-side manner, somewhat like a penguin moving across land. Thus, a chimpanzee, completely paralyzed below the neck, eventually showed recovery from a seemingly irredeemable situation. This positive outcome is thanks to the efforts of both the young volunteer care staff and the chimpanzee himself.

After recovering, Reo was occasionally visited by his mother, Reiko. Following the death of his elderly mother in 2013, his sister, Popo, took on the role of visiting her brother. The next goal is to socialize the handicapped chimpanzee so that he can live once again in the institute's chimpanzee community.
We know of one case in wild chimpanzees in which a handicapped infant was raised by her mother and big sister over about 2 years (Matsumoto et al. 2016). In the captive context, we may be able to do a lot more to help the disabled individual with the assistance of peers. The three other papers about Reo herald the development of a new discipline: "chimpanzee rehabilitation."

Please visit the following site to follow what is happening with Reo the chimpanzee, and watch a video clip of him doing the personalized touch-screen task. http://lan gint.pri.kyoto-u.ac.jp/ai/.

\section{References}

Hayashi M, Sakuraba Y, Watanabe S, Kaneko A, Matsuzawa T (2013) Behavioral recovery from tetraparesis in a captive chimpanzee. Primates 54:237-243

Matsumoto T, Itoh N, Inoue S, Nakamura M (2016) An observation of a severely disabled infant chimpanzee in the wild and her interactions with her mother. Primates 57:3-7

Matsuzawa T (2016) SAGA and GAIN for great apes. Primates $57: 1-2$

Matsuzawa T, Tomonaga M, Tanaka M (eds) (2006) Cognitive development in chimpanzees. Springer, Tokyo

Miyabe-Nishiwaki T, Kaneko A, Nishiwaki K, Watanabe A, Watanabe S, Maeda N, Kumazaki K, Morimoto M, Hirokawa R, Suzuki J, Ito Y, Hayashi M, Tanaka M, Tomonaga M, Matsuzawa T (2010) Tetraparesis resembling acute transverse myelitis in a captive chimpanzee (Pan troglodytes): long-term care and recovery. J Med Primatol 39:336-346. doi:10.1111/j. 1600-0684.2010.00415.x

Sakuraba Y, Tomonaga M, Hayashi M (2016) A new method of walking rehabilitation using cognitive tasks in an adult chimpanzee (Pan troglodytes) with a disability: a case study. Primates. doi:10.1007/s10329-016-0541-3 